\title{
Miner Face Recognition Based on Improved Singular Value Decomposition
}

\author{
JIANG Chao \\ Air Force Service College \\ XuZhou JiangSu China \\ 394952663@qq.com
}

\author{
HAN Guyong \\ Air Force Service College \\ XuZhou JiangSu China \\ yulang1980@sohu.com
}

\author{
ZHU Lin \\ Air Force Service College \\ XuZhou JiangSu China \\ zlinha@163.com
}

\author{
ZHOU Jun \\ Air Force Service College \\ XuZhou JiangSu China \\ cholgen781005@163.com
}

\author{
HUANG Weixing \\ Air Force Service College \\ XuZhou JiangSu China \\ GLXXJKS@163.com
}

\begin{abstract}
This paper depended on that video monitoring and video images in the coal mine were susceptible to dust, light and miner's safety helmet and other special environmental impact. In order to realize the real-time and accurate face recognition rate, and lay good foundation for miners' behavior characteristics in the subsequent research in intelligent video monitoring coal mine. The inherent degeneration 、 stability and Rotational invariance of singular value can reflect the matrix characteristics fully. In face recognition singular value in image matrix as miner characteristics is a very good method. But only it doesn't work using singular value of image as miners face recognition feature, aiming at face recognition, this paper combined partial singular value decomposition and the overall singular value decomposition to suit for miner image face recognition algorithm. This page Improved the previous singular value decomposition algorithm, First The singular value is decomposed in the standard characteristic matrix and projected to get a new algebraic features, Second combined the partial singular value and the overall singular value decomposition to pick up characteristics algorithm, so as to extract and reflect the mine workers face image accurate algebra feature effectively, And by using BP neural network classifier to distinguish recognition, simulation experiments prove that this method of the recognition rate is higher than other methods. The results show that the method combining the part singular value decomposition with the whole singular value decomposition can be faster, more accurate to realize face recognition, and have satisfactory recognition rate.
\end{abstract} image

Keywords-Face recognition; Miner face; singular value; video

\section{INTORDUCTION}

Coal is main energy in our country, it accounts for about $70 \%$ in non_renewable energy, and the coal-dominated energy fundamental change does not occur within the next 50 years. But In recent years, the situation about which safety production is very serious in the coal mine enterprises, heavy, serious accidents are frequent occurrence, so they cause huge losses for the property of the State and the miners' lives. This article is part of studying intelligent video surveillance in coal mine, but it is particularly important prerequisite, about face detection algorithm research based on AdaBoost algorithm in coal miner. The core of Smart video monitoring system is the detection, and track, and recognition people face and on its acts for understands and description that mainly uses computer Visual technology from video image, however the face detection and track face are important links, on acts of miners can be smart analysis and ahead of warning on exception situation, therefore, it can reduce occurs rate of accident, also guarantees production safety for coal mine [1]. Extracting miners face feature is a basic and important link for face recognition technology and looking for effective feature is the crux of dealing with miners face recognition problem.

\section{EXTRACTING FEATURE BASED ON PROJECTION TRANSFORM SINGULAR VALUE}

To descript an image matrix may use singular value characteristics. That is to say gray numerical characteristics information can be used by singular value characteristics to be described effectively [2]. This can be used to describe gray matrix of numerical characteristics. But image singular value characteristics can't be effective for miners face image recognition, because it contains only a small part of face recognition information, and a lot of important and useful information for image recognition is contained in the two orthogonal matrixes in the $\mathrm{U}$ and $\mathrm{V}$ by singular value decomposition of. According to image projection transformation is not the same (selected image reference not the same), therefor, miners face recognition is impossible to get satisfactory effect. To solve problem of the singular value characteristics can't contain a large number of important information of relevant identification characteristics, Firstly, the miner face images were put on the same standard matrix to get a new overall algebra feature based on projection coefficient. And this was used as a picture of miners face image feature vector.

Set the singular value decomposition of the standard of the face is $A=U \Lambda V^{T}, U=\left[u_{1}, u_{2}, \cdots u_{m}\right], V=\left[v_{1}, v_{2}, \cdots v_{m}\right]$,

The Article Supported by National Natural Science Foundation of China (No. 51204186) 
and the face $X$ can be expressed as in $A$ characteristic matrix projection[7]:

$$
\begin{gathered}
W_{X}=U^{T} X V=\left(\begin{array}{ccc}
u_{1}{ }^{T} X v_{1} & \cdots & u_{1}{ }^{T} X v_{m} \\
\vdots & \cdots & \vdots \\
u_{m}{ }^{T} X v_{1} & \cdots & u_{m}{ }^{T} X v_{m}
\end{array}\right) \\
\sigma=\left[u_{1}^{T} X v_{1}, u_{2}{ }^{T} X v_{2}, \cdots, u_{m}{ }^{T} X v_{m}\right] \quad \text { was showed }
\end{gathered}
$$

matrix $\mathrm{X}$ about A projection features vector, $\sigma$ was called about A projection feature vector about $B$.That is the matrix $\mathrm{W}$ elements of the diagonal. Research showed that image projection after operation can maintain its energy is changeless, However, most important information for miners face image were included in the matrix. Most of the energy was mainly distributed the array $W_{X}$ in the top left corner of the array $W_{X}{ }^{K}$. Its expression is [3]:

$$
W_{X}{ }^{K}=\left(\begin{array}{ccc}
u_{1}{ }^{T} X v_{1} & \cdots & u_{1}{ }^{T} X v_{k} \\
\vdots & \cdots & \vdots \\
u_{k}{ }^{T} X v_{1} & \cdots & u_{k}{ }^{T} X v_{k}
\end{array}\right) \in R_{X}{ }^{k}
$$

The projection that $A$ based on face $X$, previous $K$ of the largest singular value of the corresponding feature vector $U^{*}\left(u_{1}, u_{2}, \cdots, u_{k}\right), V^{*}\left(v_{1}, v_{2}, \cdots, v_{k}\right)$, These features vector coefficient contains a large number of energy of image features, So in the study use $U^{*}$ and $V^{*}$ as the standard matrix for face recognition characteristics . Finally get a new algebraic feature $W_{X}{ }^{K}$, this is mainly using the treated image which was operated in the standard on the characteristic matrix. To get the full face feature to improve the face recognition rate and can reduce the projection operation dimension, In this chapter basically use previous $k \times k$ as an average face matrix projection system[4].

\section{EXTRACTING FEATURE COMBINES THE PATR AND WHOLE SINGULAR VALUES}

The conclusion was found in the study which was based on decomposition of singular value. The whole image to expression the characteristics only showed some of the overall characteristics of the image, the important details of the image were not expressed [8]; In addition, it was also found that the image which was measured if the location or the expression changes the overall gray change obviously manifested in certain gray areas but only the more obvious changed in certain gray areas [5]. To solve this problem, we can take advantage of the block approach to deal effectively with these miners face image. Processing goal iwas to identify the characteristics of some of the sub-block vector it could be most essentially described parcel identification features to reduce unnecessary false information and identify the time required to improve the real-time performance of the system as a whole, and make full use of the monitor image [9].

In this section, to make full use of the miners' face image local feature and global feature of miners face image recognition, we use each miners' most typical local features and global features such as safety cap, miner's lamp and other important features which the miners will be with during most of their working time, and with the help of these messages, we can effectively save time for face detection. And besides, most miners' face feature may be influenced by coal, light, mining equipment and other factors, so can use the gray change of miners' obvious and important characteristics such as eyes,nose,mouth and teeth as the basis of recognition.Then do geometric computing to the important characteristics and distance between the relative relation method fully based on the partial singular value and the singular value decomposition feature and BP neural network is used for training the local feature recognition system[6]. The structure diagram as shown in the "Fig.1"

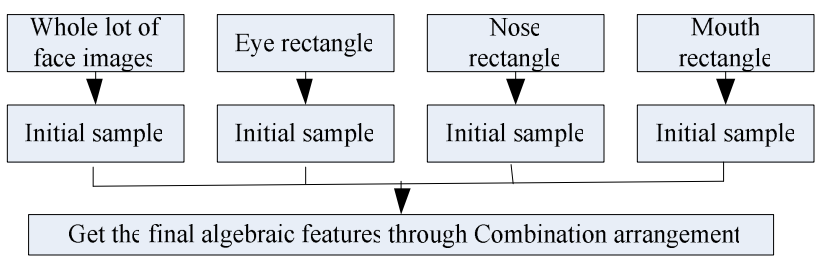

Figure1 The structure diagram of combining the part and whole singular values

\section{BASED ON PATTERN RECOGNITION CLASSIFIER OF NEURAL NETWORK}

A large number of realize show that the BP neural network has a lot of advantages, This section made full use of advantages of the adaptive ability, fault tolerant ability and parallel information processing structure, Speed and so on. So standard three layer BP neural network was chose as recognition classifier [10]. After feature extraction for miners face image to train the BP network by the characteristic vector and the corresponding teacher supervision information. As the "Fig.2" below:

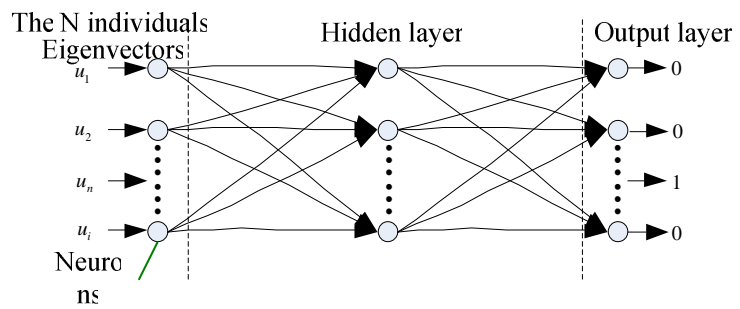

Figure2 The structure model of face recognition and BP neural network

The main ideas of the training were based on description of feature vector which were from tested faces. According to each feature vector corresponding to the input just the corresponding output was 1andothers were 0 . Used the principle of competition mechanism in the neural network to find the corresponding sample category.

Above knowable, using BP network training algorithm can be described as simple as follows:

(1)Neural network initialized weights should keep between some random number;

(2) Input monitoring image miners image acquisition as 
the training sample and calculation the training sample expectations;

(3) Stepwise calculation output;

(4) According to the result of calculation of the output from the output layer gradually began to modify the corresponding weights and error;

(5) If error is not less than the Set value, then jump to the three steps, continue to learn, otherwise, the end.

If the BP neural network can reach the stable state after training in the end then save the BP neural network, used for subsequent face recognition.

\section{TESTING RESULT AND ANALYSIS}

First of all, we collected 420 face images, a total of five groups image library, "Fig.3" is shown as one group. Each group included front and side miners face image. Used for study of face detection system, this can validate the effectiveness of the algorithm. Miners face images were collected through the artificial persons. All of the following test results are in I3-3450, $\mathrm{C}++$ and OPenCV, WinXP of the PC with 2G.
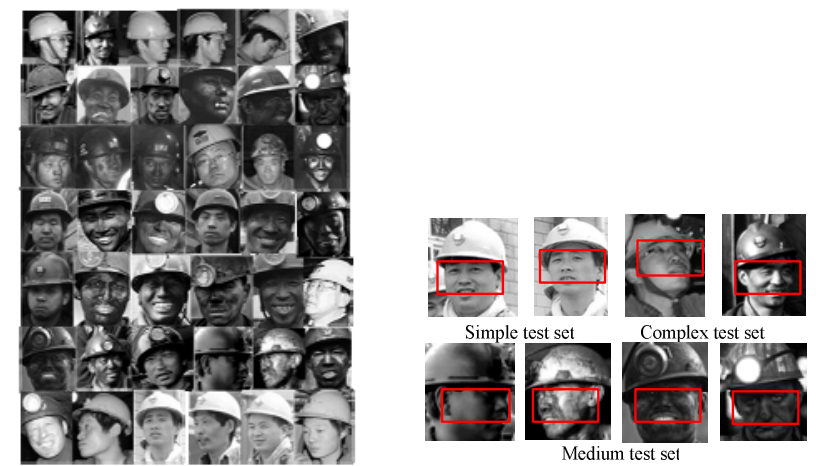

Figure $\approx$ The database of miners fac $\epsilon$ Figure4 Tested parting result of face detection

In the face detection stage AdBoost algorithm was used, this paper put the miners face samples were divided into three grades, respectively for simple test grade medium complex test grade and complex test grade, as "Fig.4" showed.

\section{A The program of the miners'face recognition}

Each miners image according to the fourth chapter was based on an improved Adaboost algorithm to detect the miners face, due to the target image could not consist with the being tested image in size and the proportion, so tested images need to be operated related preprocessing to get gray image with size $20 * 20$. According to "Fig.2" network training to obtain the characteristics of face image model library, then according to this section to combine the miners face of the features and the overall feature extraction to get singular value, according to the characteristics of the singular value decomposition by taking advantage of its former 16 Algebra features as BP neural Input for training and get recognition results. "Fig.5” is System framework of the face recognition.

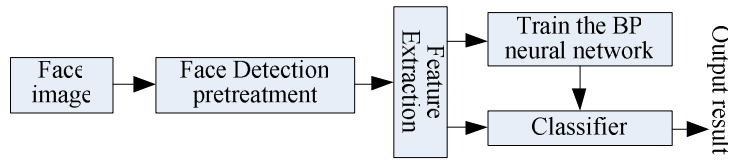

Figure5 System framework of the face recognition

\section{B Analysis testing result}

Because this system is for the miners face image, according to face image database based on the miners, the page only based on local (eyes, nose and mouth) SVD+BP Neural network, based on the technology of the whole SVD+BP neural network method, and which was based on the part and the whole SVD+BP neural network. Three miners face recognition algorithm has carried on the simulation experiment, Three related parameters of the comparison results such as table I showed:

Table I Compared different methods about correct recognition rate

\begin{tabular}{|c|c|}
\hline Face recognition method & Test recognition rate \\
\hline Part of SVD+BP neural network & $85.1 \%$ \\
\hline $\begin{array}{c}\text { Whole of SVD+BP neural network } \\
\text { Part and whole SVD+BP neural network (used } \\
\text { in this page) }\end{array}$ & $78 \%$ \\
\hline
\end{tabular}

Based on the three different methods to simulate experimental analysis, from the results of the simulation experiment table, this paper based on the value of combining feature extraction method, and combined with BP neural network mode apparatus for training identification, obtained satisfactory recognition rate, the experiment rate reached $91.8 \%$, the recognition rate is still very satisfactory, the result indicates that it is a kind of useful method.

\section{SUMMARY}

This article showed that the original singular value decomposition method was improved, on the one hand, to get the new algebra feature by projection on standard feature matrix, on the other hand to extraction algorithm combined the partial and the whole singular value decomposition. This extracts effectively the most effective algebra characteristics, and using BP neural network classifier classification to recognition, The results showed that recognition rate is higher than ordinary methods. But due to the influence of various factors for face recognition, we can consider lots of features fusion methods, and improve the recognition rate.

\section{ACKNOWLEDGMENT}

I thank the sponsor, they are Lanzhou University, Co-Sponsored by Henan University, Yan Shan University, Jiangsu University, Wenzhou University, Hebei University, Jiangsu University of Science and Technology and Xi'an University of Science and Technology.

\section{REFERENCES}

[1] ZHU Aichun, HUA Gang, WANG Yongxing. The research on the detection Method of belt deviation by video in coal mine[J].. 2011 IEEE International Conference on Mechatropic Science, Electric Engineering 
and Computer (MEC2011), 2011, 430-433.

[2] ZHU Aichun, HUA Wei, WANG Chun, WANG Yongxing. Research on the measurement of belt speed by video in coal mine based on improved template matching algorithm[J].. Journal of Coal Science \& Engineering (China), 2011, 17(4): 469-474.

[3] Yong Xing Wang, Xiu Zhu Jiang. Face detection algorithm is based on the Coal mine video monitoring image[J]. Science Paper Online.2012.1

[4] recognition[J]. Pattern Recognition Letters, 2006,27(6):609-617.

[5] Gang Hua, Lei Zhou. Attention Computation Model for Coal-mine Surveillance Video Based on Non-uniform Sampling in Spatial Domain and Time Domain[J]. PrimeAsia 2010, 107-110. (2011596513)

[6] Hua Gang, Wang Hong-Yao.Improving SNR of MFL signal in flaw detection of coal mine wire ropes[J]. CISP 2009, 2009.9 (20100212631681).
[7] Ming Wang. Face Recognition Based on Whole and Part Singular Value Decompose[J]. Control and Automation Publication Group. 2009. 07-2-0203-02(25).203-204.

[8] D. CAI, X. HE, Y. HU,et al. Learning a spatially smooth subspace for face recognition[C]. IEEE Conference on Computer Vision and Pat-tern Recognition,2007,1-8,650-656.

[9] T. KIM, J. KITTLER, AND R. CIPOLLA. Learning Discriminative Canonical Correlations for Object Recognition with Image Sets[J].Lec-ture Notes in Computer Science, 2006,3953:251.

[10] T. KIM, J. KITTLER, AND R. CIPOLLA .Discriminative Learning and Recognition of Image Set Classes Using Canonical Correlations[J]. IEEE Transactions on Pattern Analysis and Machine Intelligence, 2007, 1005-1018. 